\title{
Erratum to: Gastrointestinal stromal tumors (GISTs): SEAP- SEOM consensus on pathologic and molecular diagnosis
}

\author{
J. Martin-Broto ${ }^{1} \cdot$ V. Martinez-Marín ${ }^{2} \cdot$ C. Serrano ${ }^{3}$ N. Hindi ${ }^{1} \cdot$ J. A. López-Guerrero $^{4}$ \\ M. Biscuola ${ }^{1}$ R. Ramos-Asensio ${ }^{5}$ A. Vallejo-Benítez ${ }^{6}$ - D. Marcilla-Plaza ${ }^{7}$. \\ R. González-Cámpora ${ }^{6}$
}

Published online: 30 January 2017

(C) Federación de Sociedades Españolas de Oncología (FESEO) 2017

\section{Erratum to: Clin Transl Oncol DOI 10.1007/s12094-016-1581-2}

Due to an unfortunate error, Michele Bisculoa was omitted from the list of authors in the original article. The correct authorship of this article is as follows:

Javier Martin-Broto

Virginia Martínez-Marín

César Serrano

\author{
Nadia Hindi \\ José Antonio López-Guerrero \\ Michele Biscuola \\ Rafael Ramos-Asensio \\ Ana Vallejo-Benítez \\ David Marcilla-Plaza \\ Ricardo Gonzalez-Cámpora.
}

The competing interests section remains unchanged, since the current statement is also valid for the new author.

The online version of the original article can be found under doi:10.1007/s12094-016-1581-2.

J. Martin-Broto

jmartin@mustbesevilla.org

1 Instituto de Biomedicina de Sevilla, IBIS, Medical Oncology, Virgen del Rocío University Hospital, Seville, Spain

2 Medical Oncology, La Paz University Hospital, Madrid, Spain

3 Sarcoma Translational Research Laboratory, Vall d'Hebron Institute of Oncology (VHIO), Vall d'Hebron University Hospital, Barcelona, Spain

4 Laboratory of Molecular Biology, Fundación Instituto Valenciano de Oncología, Valencia, Spain

5 Pathology Department, Son Espases University Hospital, Palma de Mallorca, Spain

6 Pathology Department, Virgen Macarena University Hospital, Seville, Spain

7 Pathology Department, Virgen del Rocío University Hospital, Seville, Spain 